\title{
Prevalence of pulmonary embolism in patients with COVID-19 at the time of hospital admission and role for pre-test probability scores and home treatment
}
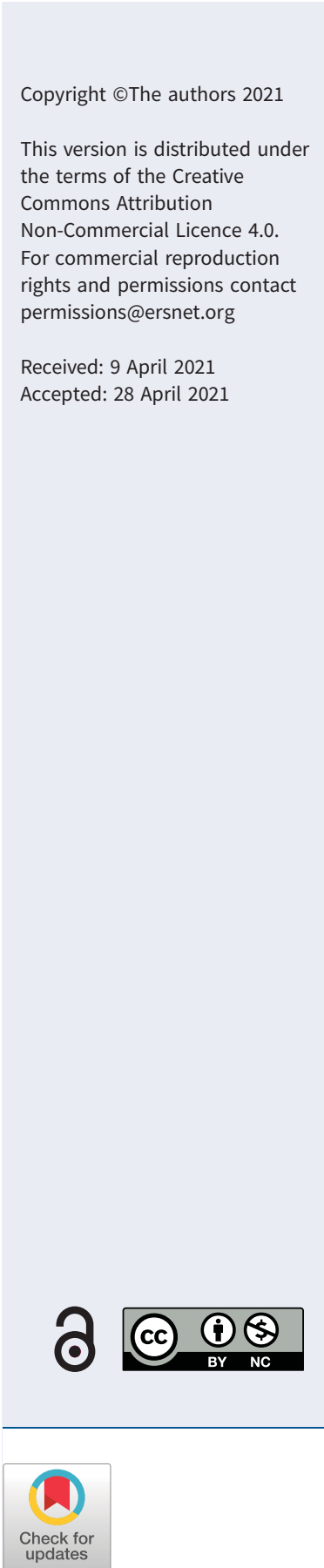

Reply to A. Porfidia and co-workers:

We thank A. Porfidia and co-workers for their interest in our study describing the prevalence of pulmonary embolism (PE) in patients with coronavirus disease 2019 (COVID-19) at the time of hospital admission [1]. We fully agree on the need to consider a PE diagnostic algorithm in COVID-19 at the time of hospital admission, because indiscriminate execution of computed tomography pulmonary angiography (CTPA) cannot be a workable, routine approach in such patients.

In order to address A. Porfidia and co-workers' question, we have collected data on risk factors for venous thromboembolism (VTE) as well history of VTE, oestrogen treatment, prior history of cancer or active cancer and surgical intervention or immobilisation in the past month. Moreover, the YEARS clinical probability for PE was prospectively studied in the emergency department by the physician in charge of the patients before knowing the results of D-dimer testing, with the application of the three items of the YEARS algorithm (clinical signs of deep vein thrombosis, haemoptysis, and PE as most likely diagnosis) [2]. Of note, the YEARS clinical probability and D-dimer results were not used to guide whether or not to perform CTPA, because all patients had CTPA at admission in our study. Nevertheless, we have been able to retrospectively evaluate the performance of the YEARS approach and the number of PEs that would have been missed, using the $1000 \mathrm{ng} \cdot \mathrm{mL}^{-1}$ D-dimer cut-off in patients without YEARS items or $500 \mathrm{ng} \cdot \mathrm{mL}^{-1}$ in patients with one or more YEARS items to exclude PE at admission, and therefore the number of CTPA that would have been avoided with this approach. Due to the word limit allowed in a research letter, we could not present these results in our original publication and we thank our colleagues for giving us the opportunity to present them in response to their correspondence.

Of the 106 included patients, 31\% (95\% CI 21.4-39.9\%) had risk factors for PE and only $16.0 \%$ of patients (95\% CI 9.6-24.4\%) had $\geqslant 1$ YEARS items, as compared to 49.7\% of the patients included in the YEARS study [2]. The YEARS algorithm was applied to the 98 patients with an available D-dimer assay. Among them, PE was diagnosed in 13 patients (13.3\%). The application of the YEARS diagnostic algorithm would have avoided 39/98 CTPA (39.8\%, 95\% CI 30-50.2\%) at the cost of missing one patient with PE (2.6\%). Briefly, this 81-year-old patient had a D-dimer level of $550 \mathrm{ng} \cdot \mathrm{mL}^{-1}$ and no YEARS items. CTPA on admission showed a distal, sub-segmental PE. Interestingly, the patient was treated with prophylactic anticoagulant treatment at home for a history of VTE, with oral apixaban $2.5 \mathrm{mg}$ twice a day. There was no significant difference between the groups with or without acute PE at the time of hospital admission for COVID-19, in terms of VTE risk factors and YEARS items $\geqslant 1$ (table 1). Finally, we confirm that no patient received COVID-19 therapy, such as corticosteroid or anticoagulation, prior to hospitalisation [1, 3].

In conclusion, determining the probability of PE is challenging in COVID-19, and a validated and safe diagnostic algorithm would be useful for clinicians. Several diagnostic algorithms have been validated and recommended in outpatients with clinical suspicion of PE but none of these algorithms has been evaluated in patients with COVID-19. The YEARS algorithm was designed to be applied in a busy clinical practice, such as that experienced in hospitals during the COVID-19 pandemic period and, for this reason, we decided to test it rather than Pulmonary Embolism Rule Out Criteria (PERC) rules [4]. Applying the

Shareable abstract (@ERSpublications)

There is a high prevalence of pulmonary embolism in patients with COVID-19 at the time of hospital admission despite a low level of clinical suspicion https://bit.ly/3ungLrQ

Cite this article as: Jevnikar M, Sanchez O, Humbert M, et al. Prevalence of pulmonary embolism in patients with COVID-19 at the time of hospital admission and role for pre-test probability scores and home treatment. Eur Respir J 2021; 58: 2101033 [DOI: 10.1183/13993003.01033-2021]. 
TABLE 1 YEARS items and risk factors for venous thromboembolism (VTE) in patients with coronavirus disease 2019 at the time of hospital admission

\begin{tabular}{|c|c|c|c|c|}
\hline & $\begin{array}{l}\text { All patients } \\
(\mathrm{n}=106)\end{array}$ & $\begin{array}{l}\text { Patients with PE } \\
\quad(n=15)\end{array}$ & $\begin{array}{l}\text { Patients without } \\
\text { PE ( } n=91)\end{array}$ & p-value \\
\hline Patients with risk factors for VTE $\mathrm{n}(\%)$ & $33(31)$ & $8(53)$ & $25(27)$ & 0.1 \\
\hline Malignancy/history of malignancy $n(\%)$ & $16(15)$ & $2(13)$ & $14(15)$ & \\
\hline $\begin{array}{l}\text { Immobilisation/surgery in the past } \\
4 \text { weeks } \mathrm{n}(\%)\end{array}$ & $13(12)$ & $2(13)$ & $11(12)$ & \\
\hline History of VTE n (\%) & $8(7)$ & $3(20)$ & $5(5)$ & \\
\hline Oestrogen $\mathrm{n}(\%)$ & $2(2)$ & $1(6)$ & $1(1)$ & \\
\hline YEARS items $\geqslant 1 \mathrm{n}(\%)$ & $17(16)$ & $5(36)$ & $12(13)$ & 0.08 \\
\hline
\end{tabular}

YEARS algorithm in our study population would have allowed to avoid almost 40\% of CTPA at hospital admission at the cost of one false-negative (2.6\%). However, these data must be interpreted with caution because of the small number of patients studied. Larger prospective studies are needed to validate the value of PE diagnostic algorithms in that challenging clinical setting also due to the high PE prevalence despite a low level of clinical suspicion at hospital admission [1].

Mitja Jevnikar ${ }^{1,2,3}$, Olivier Sanchez ${ }^{4,5,6,7}$, Marc Humbert $\oplus^{1,2,3}$ and Florence Parent ${ }^{1,2,3}$

${ }^{1}$ Université Paris-Saclay, Faculty of Medicine, Le Kremlin-Bicêtre, France. ${ }^{2}$ INSERM UMR_S 999, Le Kremlin-Bicêtre, France. ${ }^{3}$ AP-HP, Service de Pneumologie et soins intensifs respiratoires, Hôpital Bicêtre, Le Kremlin-Bicêtre, France. ${ }^{4}$ AP-HP, Service de Pneumologie et Soins Intensifs, Hôpital Européen Georges Pompidou, Paris, France. ${ }^{5}$ INSERM UMR-S 1140, Paris, France. ${ }^{6}$ INNOVTE, StEtienne, France. ${ }^{7}$ Université Paris Descartes, Faculty of Medicine, Paris, France.

Corresponding author: Florence Parent (florence.parent@aphp.fr)

Conflict of interest: M. Jevnikar has nothing to disclose. O. Sanchez reports personal fees and non-financial support from BTG, grants and personal fees from Boston Scientific, grants, personal fees and non-financial support from Bayer and BMS, personal fees from Sanofi Aventis, grants from Daiichi Sankyo, during the conduct of the study; grants, personal fees and non-financial support from MSD, grants from Boehringer Ingelheim, outside the submitted work. M. Humbert reports grants and personal fees from Actelion, personal fees from GSK and Merck, outside the submitted work. F. Parent has nothing to disclose.

\section{References}

1 Jevnikar M, Sanchez O, Chocron R, et al. Prevalence of pulmonary embolism in patients with COVID-19 at the time of hospital admission. Eur Respir J 2021; 58: 2100116.

2 Van der Hulle T, Cheung WY, Kooij S, et al. Simplified diagnostic management of suspected pulmonary embolism (the YEARS study): a prospective, multicentre, cohort study. Lancet 2017; 390: 289-297.

3 Chalmers JD, Crichton ML, Goeminne PC, et al. Management of hospitalised adults with coronavirus disease 2019 (COVID-19): a European Respiratory Society living guideline. Eur Respir J 2021; 57: 2100048.

4 Penaloza A, Soulié C, Moumneh T. Pulmonary embolism rule-out criteria (PERC) rule in European patients with low implicit clinical probability (PERCEPIC): a multicentre, prospective, observational study. Lancet Haematol 2017; 4: e615-e621. 